\title{
Neural network forecast of the solar, geomagnetic activity and cosmic ray intensity during 25 cycle
}

\author{
Zbigniew Kobylinski ${ }^{1}$ \\ High School of National Economics in Kutno \\ Kutno, Poland \\ E-mail: zbigniewkobylinski@yahoo.com
}

\section{Arkadiusz Wysokinski \\ Independent researcher \\ Iganie, Poland \\ E-mail: arekwysetlen.pI}

\section{Tomasz Seredyn}

Polish Air Force University,

Deblin, Poland

E-mail: t.seredyn Qlaw.mil.pl

\section{Abstract}

The purposes of the paper are at first to inspect the solar and geomagnetic variations during the solar cycle 24 and the earlier cycles from $16^{\text {th }}$ and the second to calculate the solar number progression during the next two solar cycles basing on the earlier cycles data from the period 1923 - 2019 by means of the artificial neural networks (ANN). We focus our attention on Elman ANN because comparisons of an ANN type effectiveness in modeling of disturb course of different solar and geomagnetic parameters indicated on an recurrent ANN as better predictor than equivalent feedforward ANN. Daily data averaged by Bartels rotation of different activity parameters as sunspot numbers, aa geomagnetic indices, neutron monitor records from a station ROME with cut-off rigidity of $6.24 \mathrm{GV}$ are used. Because of the limited place for this report our estimations related to variability of cosmic ray intensity would be presented only in the poster. Additionally the wavelet technique is used to show the coherence between estimated data. We compare the results and estimate that the solar activity would be rather a little smaller during 25 cycle than in 24 cycle.

\section{Introduction}

Solar activity is not a stationary process, it is best described as quasi-stationary and quasi-periodic. The behavior depends upon the time interval under consideration. Nowadays we have long time series of solar parameters and we can make efforts to forecast the future phenomena owing to long term observations. In this paper we briefly compare the recent solar variability and geomagnetic field changes with earlier

\footnotetext{
Presenter Tomasz Seredyn
} 
behavior basing on two indices: sunspot number $\left(\mathrm{R}_{\mathrm{i}}\right)$, aa index and ROME station data from WDC for Cosmic Rays at Nagoya University. Both the first parameters used are taken from the NOAA websites (http://www.ngdc.noaa.gov). In the second part of the paper we show the results of predictions for solar cycles 25/26.

\section{Solar and geomagnetic changes during cycles $16-24$.}

The last solar cycle 24 started in Dec 2008 as it is pointed out by smoothed monthly mean sunspot number equal 1.7 . Approximately at the same time of minimum the minimal value of aa index about 8 was calculated. From cycle 16 till nowadays values of Bartels rotation means of sunspot number and aa index are presented in Fig.
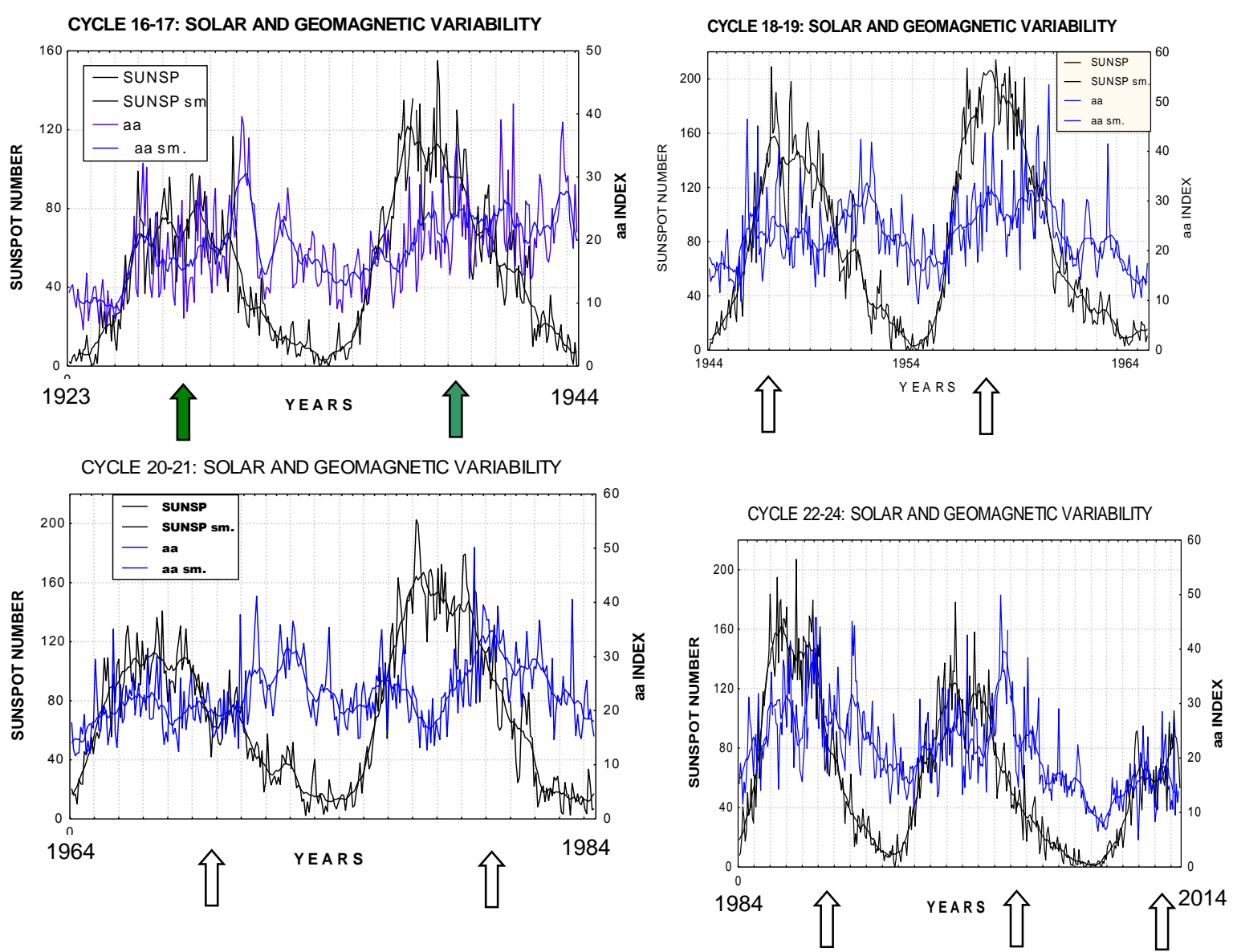

Figure 1. Solar and geomagnetic variability during cycles 16 - 24 (Bartels rotation 1238 - 2468). Both indices are averaged by Bartels rotation, also 11-point moving means are shown. Arrows polar field reversals: green - estimated, empty - real onces.

The amplitude of 24 cycle is similar to solar cycle 16 . Both cycles are smallest during the period of the last 100 years. Sunspot maxima well reflect a part of Gleissberg period of $\approx 80$ years. We could expect a small growth of activity in cycle 25 and in the next in the time scale of tenth years. Similar behavior as sunspot numbers is 
observed in other solar indices, as it has been shown by Kane (2014) $)^{2}$. The first maximum of sunspot numbers occurred in Oct 2011 (Bart. rot. 2432) =91,6, the next peak of activity happened in May 2012 (Bart. rot. 2440) = 71,3, the third in Apr 2013 $($ Bart. rot. 2452) $=73,1$, the fourth in Feb 2014 (Bart. rot. 2463) $=105.1$. The similar complex pattern of the period of maximal activity was observed during cycle 16 and in cycle 17 but with higher values of $\mathrm{R}_{\mathrm{i}}$. It could be connected with polar field reversal: the northern polar field of the Sun reversed in June 2012, the southern polar field reversed in Sep 2013. Geomagnetic activity delayed one or two years practically in every cycle. Patterns of geomagnetic variability are more complex than solar one.

\section{Solar and geomagnetic activity predictions for cycles 24.}

Solar activity prediction is very important in space weather studies. Prediction of the parameters of the solar cycle is significant for planning satellite launching, as large eruptions of solar plasma and solar cosmic rays can cause damage and malfunction of satellite and aircraft electronics and health hazards in space, as well as can spoil radiobased communication and navigation systems. Also there are possible connections between solar activity, clouds and climate through influences of galactic cosmic ray flux and solar irradiance onto cloud cover data, see Svensmark and Friis-Christensen $(1997)^{3}$, Kristjansson, Staple and Kristiansen (2007) ${ }^{4}$. For instance it is believed that the main course of the Little Ice Age near the years 1600 - 1700 was caused by reduced solar irradiance [Lean and Rind $(1998)^{5}$ ]. By turns assumptive global warming is important for bankers and is the base for their financial machinations .

A number of techniques have been developed to predict the amplitude of solar maximum, duration and shape of a following solar cycle. They have changed from simple statistical to complex physical, as for example geomagnetic precursor, based on the understanding of the dynamo process that generates the solar magnetic field and its evolution. Summary lists of predictions of the last solar cycle 24 were given for example by Janssens (2005) ${ }^{7}$ and Pesnell (2008) ${ }^{8}$. The big disaccord of prediction results could be pointed out. The variability from one cycle to the next observed in sunspot, and especially in geomagnetic records, generates the difficulty in making empirical predictions.

\footnotetext{
2

Kane R.P. (2014). Evolution of solar indices during the maximum of solar cycle 24. Indian J. Radio \& Space Phys v. 43, 151-155.

Svensmark H., Friis-Christensen E. (1997). Variation of cosmic ray flux and global cloud coverage: A missing link in solar climate relationships. J. Almos. Solar-Terr. Phys., 59, 1225-1232.

Kristjansson J. E., Staple A., and Kristiansen J. (2007). A new look at possible connections between solar activity, clouds and climate. Geophys. Res. Lett. v. 29, no. 23, 22-1-22-4.

Lean J., Rind D. (1998). Climate forcing by changing solar radiation. J. Climate, v. 11, 3069-3094.

von Retyi A. (2016). George Soros. Der Multimilliardar, sein globales Netzwerk und das Ende der Welt, wie wir sie kennen. Kopp Verlag e.K. Germany, 110-120.

Janssens J., (2005). Solar cycle 24: Overview of predictions on the start and amplitude of a new solar cycle. (http://memebers.chello.be/j.janssens/SC24pred.pdf).

Pesnell W.D. (2008). Predictions of solar cycle 24. Sol. Phys. v. 252, 209-220.
} 
From the pictures in Fig. 1 it is seen that solar activity in the last cycle 24 was much lower than in the previous cycles 21-23. The reduction of solar activity in cycle 24 led several authors to conclusion that the Sun is on its way towards the next Maunder Minimum of activity [Loockwod at all (2011) ]. In the several papers of Zharkova et all, see Scientific Reports (2015) and ref. therein , where the PCA method was applied to low-resolution full disk magnetograms from the Wilcox Solar Observatory, it was found that there were two magnetic waves of the poloidal field which could account for the observed sunspot magnetic field. The result of calculations with Euriqa software was presented in Fig. 2 at above mentioned Report and was first published by Shepherd at all $(2014)^{11}$. The modulus curve plotted for cycles 21-23 corresponds rather closely to the averaged sunspot numbers for cycles 21 and 22, but is lower for cycle 23, and significantly reduced amplitudes of the summary curve for cycles 25-27 or the next predicted Maunder Minimum. Thus, accordingly to these authors solar activity during such period will be extremely low.

\section{Elman Recurent Artificial Neural Networks}

A relatively small number of papers are based on the artificial neural network (ANN) adoption to the forecast of the future solar and geomagnetic activity data sets. A neural network forecast is derived from a set of nonlinear, statistical, data-modeling algorithms. They are used to determine and model complex relationships between inputs and outputs or to find patterns in data that can be extrapolated. There are some papers in literature in which ANNs have been adopted to solve geophysical problems especially related with forecast of solar-terrestrial effects, as the solar cycle 24 prediction by means of feed-forward ANN [Maris at al., 2006) ${ }^{12}$ ], the magnetic storms and rapid changes of disturbance indices [Lundstedt (1992) ${ }^{13}$ ]

There are many different types of such networks, nevertheless we shall focus our attention on Elman ANN because comparisons of an ANN type effectiveness in modelling of disturb course of geomagnetic parameters have indicated on an recurrent predictor than equivalent feed-forward ANN [Barkhatov et al., (2000) ${ }^{14}$ ]. In Elman ANN the input consists of two parts: the proper input layer and the context one, which has a

\footnotetext{
9

Loockwod C.A., Owens M.J., Barnard L., Davis C.J., Steinhilber F. (2011). The persistence of solar activity indicatros and the descent of the Sun into Maunder Minimum conditions. Geophys. Res. Lett. v. $38,22105$. 10

Zharkova V.V., Popova E.P., Zharkov S. (2015). Heartbeat of the Sun from Principal Component Analysis and prediction of solar activity on a millenium timescale. Scientific Reports: https://www.researchgate.net/publication/283862631 11

Shepherd S.J., Zharkov S.J., Zharkova V.V. (2014). Prediction of Solar Activity from Solar Background Magnetic Field Variations in Cycles 21-23. Astrophys. J. 795, 46.

Maris G., Onica A. (2004). Solar cycle 24 forecast. Sun and Geosphere, Vol. 1(1).

Lundstedt H. (1992) Neural networks and predictions of solar-terrestrial effects, Plan. Space Sc. Vol.40, No. 4, 457-464.

Barkhatov N.A., Belliustin N. S., Levitin A.E., Sakharov S.Yu. (2000). Comparison of artificial neural network efficiency for predicting the geomagnetic activity index $\mathrm{D}_{\mathrm{st}}$. Izvestia Vuzov, Radiofizika, XVII, vol. 5,385-394 (in Russian).
} 


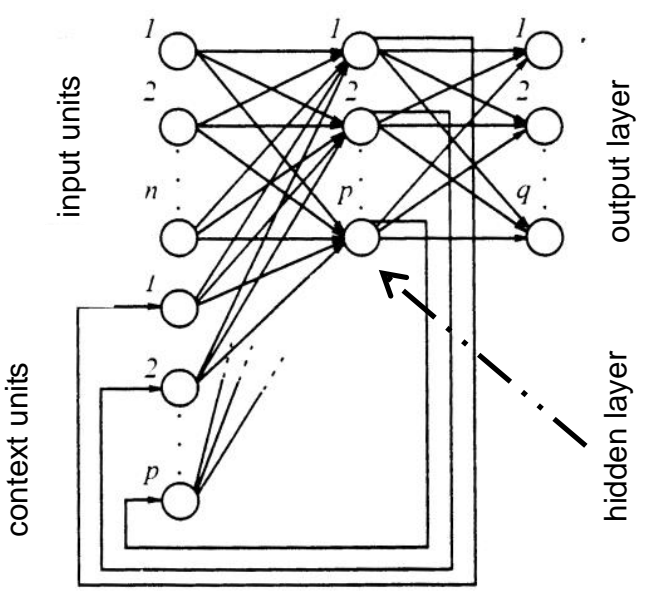

Fig.2. Elman neural networks architecture.

feedback connection with the hidden units with the fixed weights of $1.0^{15}$. Owing to these values of the weights at each step the exact information from output of hidden neurons are copied to context unit. So, the context neurons remember the previous state. Accordingly to this feature the Elman ANN is especially useful in time series analysis and for predictions of effects which are depended on time.

The architecture of the Elman ANN is showed on Fig. 2 where $n$ is number of inputs, $p$ is the number of neurons in hidden and context layer, $q$ is a number of output nodes. The hidden layer is directly feed-forward connected to the output unit. The transfer function of neurons in the hidden layer is the hyperbolic tangent and at output layer is the linear function.

Let the vector of input data is $\mathbf{I}=\left(I_{1}, ., I_{n}\right)$, the vector of values from the output of hidden layer is $\mathbf{V}=\left(\mathrm{V}_{1}, . . \mathrm{V}_{\mathrm{p}}\right)$ and the vector of output signal is $\mathbf{Y}=\left(\mathrm{Y}_{1}, ., \mathrm{Y}_{\mathrm{q}}\right)$. At the step of repetition of calculation $t$ value on the output of hidden neuron enumerated by $j$ is equal

$$
\mathrm{V}_{\mathrm{j}}(\mathrm{t})=\tanh \left[\sum_{\mathrm{k}=1}^{\mathrm{n}} \mathrm{W}_{\mathrm{jk}} \mathrm{I}_{\mathrm{k}}(\mathrm{t})+\sum_{\mathrm{k}=\mathrm{n}+1}^{\mathrm{n}+\mathrm{p}} \mathrm{W}_{\mathrm{jk}} \mathrm{V}_{\mathrm{k}}(\mathrm{t}-1)\right],
$$

where $\mathrm{j}=(1, \ldots, \mathrm{p})$, where $\mathrm{V}_{\mathrm{k}}(\mathrm{t}-1)$ is the value at one calculation step earlier, $\mathrm{W}_{\mathrm{jk}}$ are the weights of connections between the input and hidden layers. The desired result value from the output node $\mu$ at the step $t$ is following

$$
\mathrm{Y}_{\mu}=\sum_{\mu=1}^{\mathrm{p}} \mathrm{W}_{\mu \mathrm{j}} \mathrm{V}_{\mathrm{j}}(\mathrm{t})
$$

where $\mu=(1, ., q)$. The cost function which estimated an error of calculation at the step $t$ is defined as

$$
\mathrm{E}(\mathrm{t})=\sum_{\mu=1}^{\mathrm{q}}\left[\mathrm{Y}_{\mu}(\mathrm{t})-\mathrm{C}_{\mu}(\mathrm{t})\right]^{2} \quad
$$

15

Elman J.L. (1990). Finding structure in time. Cognitive Science 14, 179-211. 
where $\mathrm{C}_{\mu}$ are the values determined in output.

For training the network we use the gradient descent with momentum and adaptive learning rate back propagation paradigm [Haykin, (1994) ${ }^{16}$ ]. The values of weights are adjusted on every step (epoch) of calculation by increments proportional to appropriate components of gradient vector of the cost function relative to weights calculated during the former step

$$
\Delta \mathrm{W}_{\alpha, \beta}^{\mathrm{m}}(\mathrm{t}+1)=\xi \Delta \mathrm{W}_{\alpha, \beta}^{\mathrm{m}}(\mathrm{t})+(1-\xi) \eta \nabla_{\alpha, \beta}^{\mathrm{m}} \mathrm{E}(\mathrm{t}),
$$

where $\alpha, \beta$ are numbers of neurons, $\eta$ is a learning rate, $\xi$ momentum parameter, $m$ the layer number. Parameters $\xi, \eta$ are automatically adjusted during training in order to improve the convergence.

\section{The wavelet coherence analysis}

We apply the wavelet technique which lets to estimate the coherence between analyzed time series of data. The magnitude-squared coherence between two time series $x(t)$ and $y(t)$ at the frequency $\omega$ is defined as

$$
\mathrm{C}_{\mathrm{xy}}{ }^{2}(\omega)=\left|\mathrm{P}_{\mathrm{xy}}(\omega)\right|^{2} / \mathrm{P}_{\mathrm{xx}}(\omega) \mathrm{P}_{\mathrm{yy}}(\omega),
$$

where $t$ is time, $P_{x x}$ and $P_{y y}$ are power spectral densities of the series, $P_{x y}$ is the cross spectral density between these time series [Otnes R.K., Enochson L. (1972) ${ }^{17}$ ]. The wavelet transform describes filtration of an analyzed signal $x(t)$ using real or complexvalued function $\psi(t, a)$, which is defined as

$$
\left.\mathrm{W}_{\mathrm{x}}(\mathrm{t}, \mathrm{a})=\left(1 / \mathrm{a}^{-0.5}\right) \int \psi^{*}(\tau-\mathrm{t}) / \mathrm{a}\right) \cdot \mathrm{x}(\tau) \mathrm{d} \tau,
$$

where * denotes complex conjugate, variable $a$ and $\tau$ are the dilation (frequency) and translation (position) scaling factors. In the case of one-dimensional original signal, the continuous wavelet transform generates a two-dimensional picture of the wavelet power (scalogram) [ Daubechies I. (1992) ${ }^{18}$ :

$$
\mathrm{S}_{\mathrm{x}}(\mathrm{t}, \mathrm{a})=\left|\mathrm{W}_{\mathrm{x}}(\mathrm{t}, \mathrm{a})\right|^{2} \text {. }
$$

The cross wavelet transform of two time series $x$ and $y$ is defined as

$$
\mathrm{S}_{\mathrm{xy}}(\mathrm{t}, \mathrm{a})=\mathrm{W}_{\mathrm{x}}(\mathrm{t}, \mathrm{a}) \mathrm{W}_{\mathrm{y}}(\mathrm{t}, \mathrm{a}),
$$

the wavelet coherence (WTC) of two signals or time series is defined as

$$
\mathrm{C}_{\mathrm{xy}}{ }^{2}(\mathrm{t}, \mathrm{a})=\left|\mathrm{A}\left(\mathrm{a}^{-1} \mathrm{~S}_{\mathrm{xy}}(\mathrm{t}, \mathrm{a})\right)\right|^{2} /\left(\mathrm{A}\left(\mathrm{a}^{-1}\left|\mathrm{~S}_{\mathrm{x}}(\mathrm{t}, \mathrm{a})\right|\right) \cdot \mathrm{A}\left(\mathrm{a}^{-1}\left|\mathrm{~S}_{\mathrm{y}}(\mathrm{t}, \mathrm{a})\right|\right)\right)
$$

where $A$ is a smoothing operator along time axis and scale axis. It is a complex function and is resembled the formula for correlation coefficient, $0 \leq C_{x y}{ }^{2}(t, a) \leq 1$. When coherence values for two time series are close to one it can be concluded that both data sets are very similar in a wide range of frequencies. In the paper we use the packages prepared by Torrence \& Compo (1998), Grinsted et al. (2004) and statistic program bewalet $R$, which employ the Morlet wavelet in order to evaluate scalograms

\footnotetext{
16 Haykin S. (1994). Neural networks, a comprehensive foundation. Macmillan College Publ. Comp. NY. 18

Otnes R.K., Enochson L. (1972). Digital Time Series Analysis. J. Wiley \& Sons, New York.

Daubechies I. (1992).Ten lectures on wavelets, SIAM, Philadelphia.
} 
and WTC of two data sets:

$$
\psi(\mathrm{t}, \mathrm{a})=\pi^{-1 / 4} \mathrm{e}^{\mathrm{i} \omega_{0} \eta} \mathrm{e}^{-\frac{1}{2} \eta^{2}}
$$

where $\omega_{0}$ is the dimensionless frequency, $\eta$ is the dimensionless time ${ }^{19}$.

\section{Data treatment}

In our analysis we have extracted the time period Jan 1923 - Apr 2029 (Bart. rot. 1231-2677). We firstly have used the daily data sets of sunspot number $\left(R_{i}\right)$ and geomagnetic index aa, in the second part of this research we have used cosmic ray intensity data from Jan 1939 till Dec 2016 (in Poster). They were smoothed by means of calculation of Bartels rotation means which better reflect the variability of these data than the monthly means. For the next procedure we have obtained 1308 known values for every series and we had to found 140 values for solar cycle 25. As usual practice we used 720 values as the training set of Elman ANN, 140 as the validation set and 140 as the test set. A number of hidden neurons was found through tests: 40 . Activation function: sig The mean square error automatically calculated during process of learning as a function of a number of cycles. After 500 cycles the training was interrupted as it can be treated that the target output values reached a stable minimum.

The time relation between solar and geomagnetic activity or cosmic ray intensity could be tested by means the powerful wavelet analysis and wavelet coherence scalograms. Results are presented in Tables and the next Figures.

\section{Results}
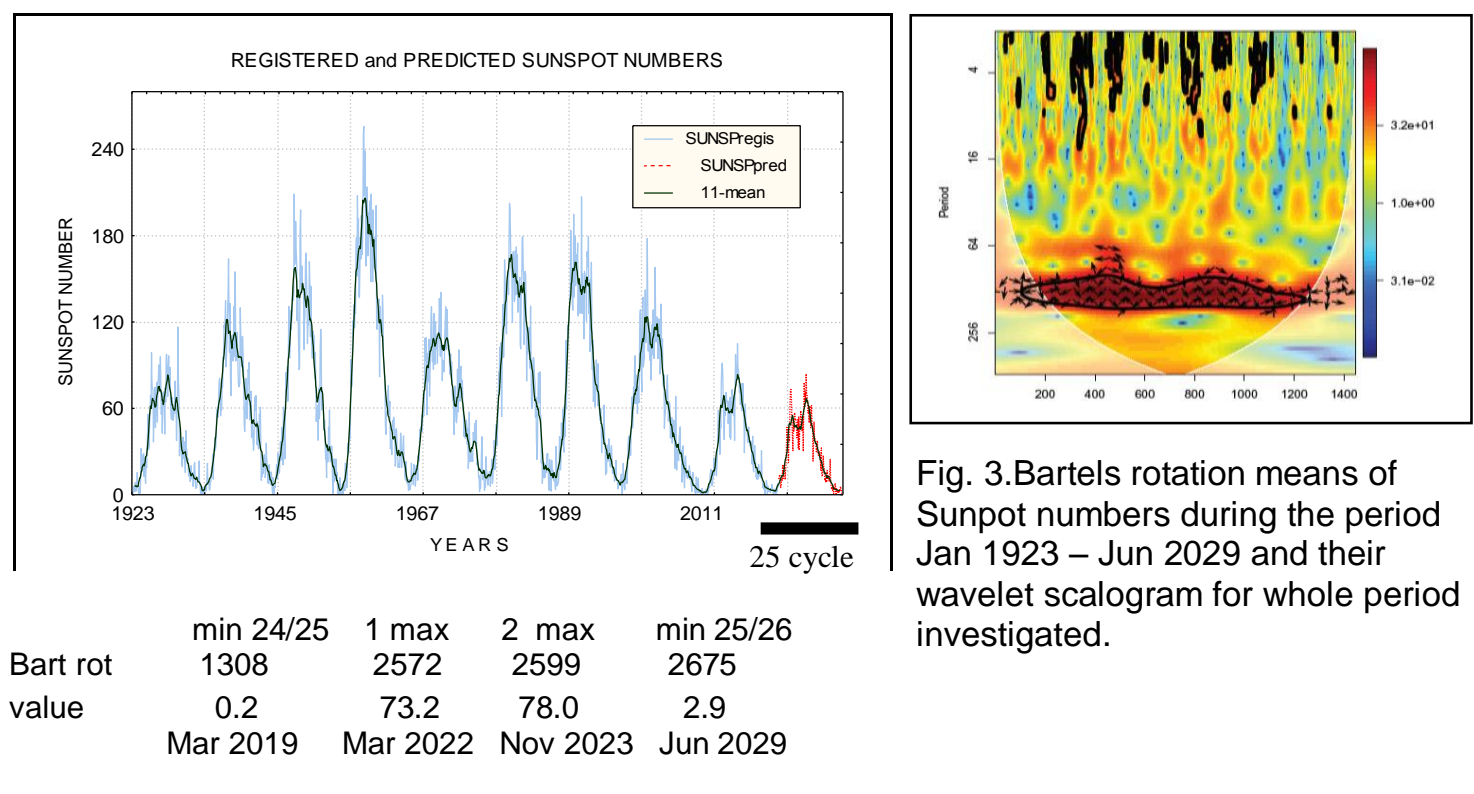

Fig. 3.Bartels rotation means of Sunpot numbers during the period Jan 1923 - Jun 2029 and their wavelet scalogram for whole period investigated.

19

Torrence C., Compo G.P. (1998). A practical guide to wavelet analysis. Bull. Amer. Meteor. Soc., 79, 61-78, (http://paos.colorado.edu/research/wavelets/; Grinsted A., J.C. Moore, S. Jevrejeva (2004). Application of the cross wavelet transform and wavelet coherence to geophysical time series. Nonlin. Proc. Geophys., v. 11, 561-566. (http://www.pol.ac.uk/home/research/waveletcoherence/ 

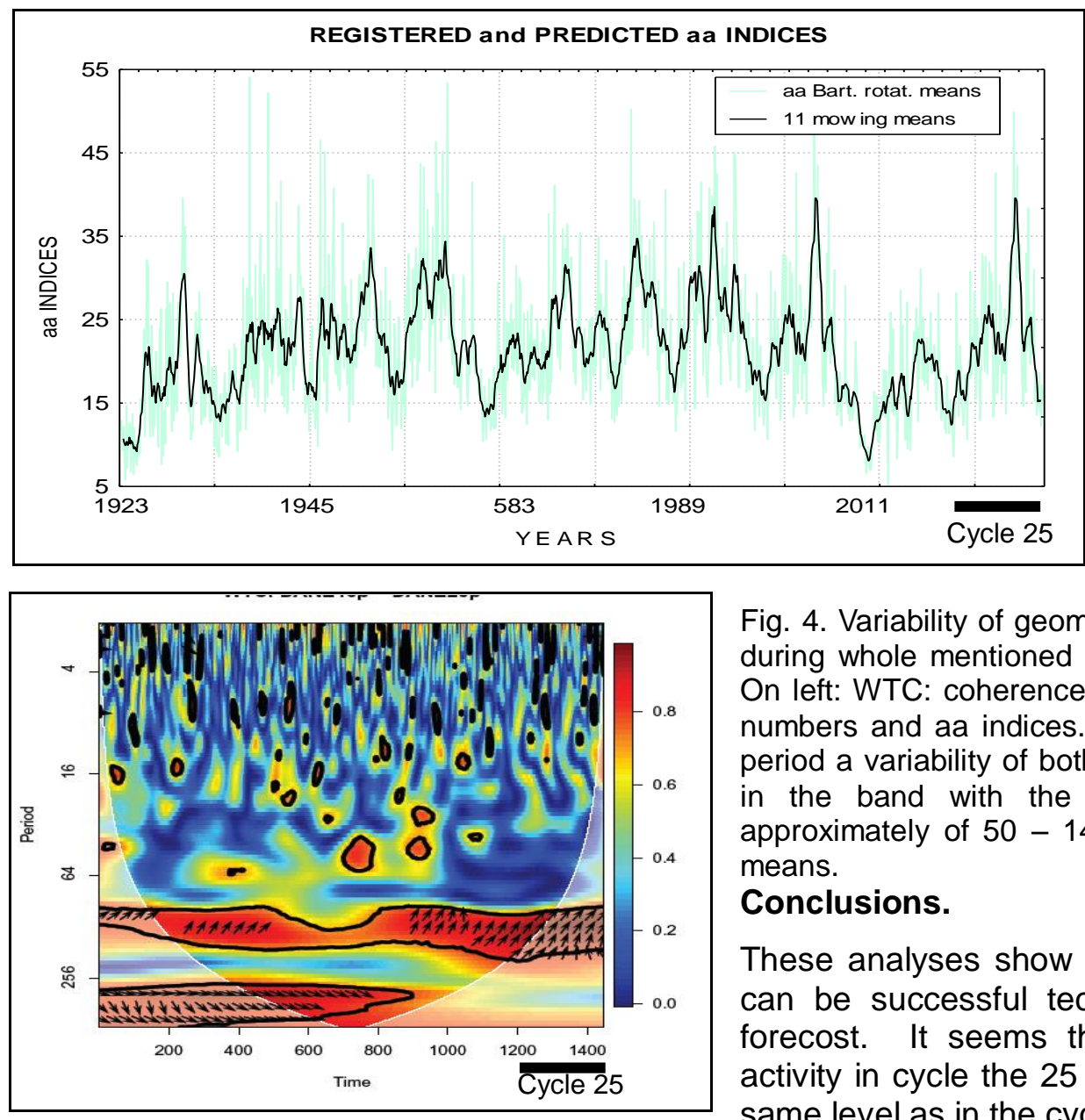

Fig. 4. Variability of geomagnetic conditions during whole mentioned period 1923-2029. On left: WTC: coherence between Sunspot numbers and aa indices. During the whole period a variability of both indices is similar in the band with the periods between approximately of $50-140$ Bartels rotation means.

\section{Conclusions.}

These analyses show that Elman ANN can be successful technique for data forecost. It seems that geomagnetic activity in cycle the 25 would be on the same level as in the cycle 24.

\section{References}

Barkhatov N.A., Belliustin N. S., Levitin A.E., Sakharov S.Yu. (2000) Izvestia Vuzov, Radiofizika, XVII, v. 5,385-394 (in Russian).

Daubechies I. (1992).Ten lectures on wavelets, SIAM, Philadelphia.

Elman J.L. (1990) Cognitive Science 14, 179-211.

Grinsted A., J.C. Moore, S. Jevrejeva (2004) Nonlin. Proc. Geophys., v. 11, 561-566.

Haykin S. (1994) Neural networks, a comprehensive foundation. Macmillan College Publ. Comp. NY.

Janssens J., (2005) (http://memebers.chello.be/j.janssens/SC24pred.pdf)

Kane R.P. (2014) Indian J. Radio \& Space Phys v. 43, 151-155.

Kristjansson J. E., Staple A., and Kristiansen J. (2007) Geophys. Res. Lett. v. 29, no. 23, 22-1-22-4.

Lean J., Rind D. (1998). J. Climate, v. 11, 3069-3094.

Loockwod C.A., Owens M.J., Barnard L., at all. Geophys. Res. Lett. v. 38, 22105.

Maris G., Onica A. (2004) Sun and Geosphere, Vol. 1(1).

Lundstedt H. (1992) Plan. Space Sc., Vol.40, no. 4, 457-464.

Otnes R.K., Enochson L. (1972) J. Wiley \& Sons, New York.

Pesnell W.D. (2008) Sol. Phys. v. 252, 209-220.

von Retyi A. (2016) George Soros. Der Multimilliardar, sein globales Netzwerk und das Ende der Welt, wie wir sie kennen. Kopp Verlag e.K. Germany, 110-120.

Shepherd S.J., Zharkov S.J., Zharkova V.V. (2014) Astrophys. J. 795, 46.

Svensmark H., Friis-Christensen E. (1997) J. Almos. Solar-Terr. Phys., 59, 1225-1232.

Torrence C., Compo G.P. (1998) Bull. Amer. Meteor. Soc., 79, 61-78.

Zharkova V.V., Popova E.P., Zharkov S. (2015) Scientific Reports:

(https://www.researchgate.net/publication/283862631) 